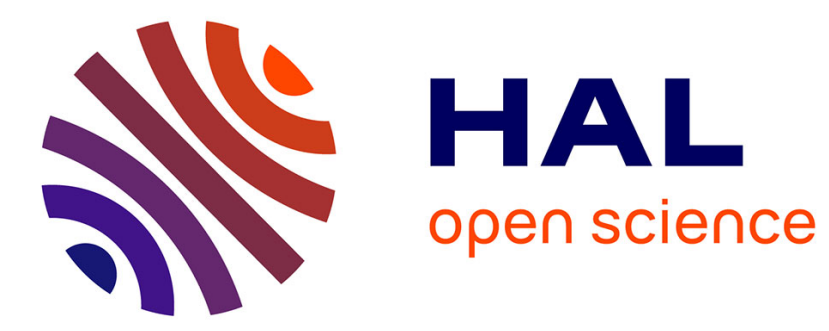

\title{
Photon Bunching in Cathodoluminescence
}

S. Meuret, L. h. g. Tizei, T. Cazimajou, R. Bourrellier, H. c. Chang, F. Treussart, M. Kociak

\section{To cite this version:}

S. Meuret, L.h.g. Tizei, T. Cazimajou, R. Bourrellier, H.c. Chang, et al.. Photon Bunching in Cathodoluminescence. Physical Review Letters, 2015, 114 (19), 10.1103/PhysRevLett.114.197401. hal-02059627

\section{HAL Id: hal-02059627 https://hal.science/hal-02059627}

Submitted on 4 Jun 2021

HAL is a multi-disciplinary open access archive for the deposit and dissemination of scientific research documents, whether they are published or not. The documents may come from teaching and research institutions in France or abroad, or from public or private research centers.
L'archive ouverte pluridisciplinaire HAL, est destinée au dépôt et à la diffusion de documents scientifiques de niveau recherche, publiés ou non, émanant des établissements d'enseignement et de recherche français ou étrangers, des laboratoires publics ou privés. 


\title{
Photon Bunching in Cathodoluminescence
}

\author{
S. Meuret, ${ }^{1}$ L. H. G. Tizei, ${ }^{1}$ T. Cazimajou, ${ }^{1}$ R. Bourrellier,${ }^{1}$ H. C. Chang, ${ }^{2}$ F. Treussart, ${ }^{3}$ and M. Kociak ${ }^{1, *}$ \\ ${ }^{1}$ Laboratoire de Physique des Solides, UMR 8502 CNRS and Université Paris-Sud, Orsay 91405, France \\ ${ }^{2}$ Institute of Atomic and Molecular Sciences, Academia Sinica, Taipei 106, Taiwan \\ ${ }^{3}$ Laboratoire Aimé Cotton, UMR 9188 CNRS, Université Paris Sud and ENS Cachan, Orsay 91405, France
}

(Received 23 December 2014; published 11 May 2015)

\begin{abstract}
We have measured the second order correlation function $\left[g^{(2)}(\tau)\right]$ of the cathodoluminescence intensity resulting from the excitation by fast electrons of defect centers in wide band-gap semiconductor nanocrystals of diamond and hexagonal boron nitride. We show that the cathodoluminescence second order correlation function $g^{(2)}(\tau)$ of multiple defect centers is dominated by a large, nanosecond zero-delay bunching $\left(g^{(2)}(0)>30\right)$, in stark contrast to their flat photoluminescence $g^{(2)}(\tau)$ function. We have developed a model showing that this bunching can be attributed to the synchronized emission from several defect centers excited by the same electron through the deexcitation of a bulk plasmon into few electron-hole pairs.
\end{abstract}

DOI: 10.1103/PhysRevLett.114.197401

PACS numbers: 78.60.Hk, 71.55.Cn, 42.50.Ar

In recent years, the emergence of nanoparticles and heterostructures with original optical properties has coincided with the development of new optical microscopy and spectroscopy techniques with subwavelength resolutions.

In this field, techniques such as scanning (transmission) electron microscopy-S(T)EM-and associated spectroscopies, are of particular interest. They benefit from the very high spatial resolution allowed by the picometer-range electron wavelength and ultimately from the spectral resolution arising through laser monochromaticity [1]. Indeed, cathodoluminescence (CL) [2] and electron energy-loss spectroscopy (EELS) [3,4] have emerged as major techniques for nanoplasmonic studies; understanding the similarities and differences between these methods and pure optical ones has led to an extremely fertile field of research $[5,6]$. On the other hand, CL in a scanning electron microscope (SEM) has long been a key technique for studying semiconducting materials [7]. Increasingly higher resolution SEMs and the emerging use of STEM-CL [8] makes CL a particularly suitable technique for nanophotonics applications [9-12]. However, contrary to the case of nanoplasmonics, the comparison between electron and photon based spectroscopies is almost unexplored in the field of nanostructured semiconductors.

When a semiconductor material is excited by a photon, the energy transfer immediately creates an electron-hole $(e-h)$ pair that decays to the energy level of the most probable radiative transition in the material. Electronmatter interactions are more complex because they give rise to a range of primary excitations, essentially plasmons and hot $e-h$ pairs, with varying energies. The number of primary excitations per incoming electron depends on the thickness of the sample and the energy of the electron. In typical STEM conditions, this number is close or even less than 1 and can be measured experimentally [13], making
STEM-CL an appealing technique for unveiling the electron-matter interaction physics.

Various theories have been proposed to account for the chain of events leading from the electron-matter interaction to $e-h$ creation $[14,15]$ with the inclusion of plasmons, by far the dominant source of primary electron energy loss $[16,17]$, as intermediate states $[18,19]$. This highlights major differences between photoluminescence (PL) and CL excitation mechanisms, although they seem to have no influence on the emission spectra, which are almost identical between both techniques [20]. Thus, the impact of plasmon decay mechanisms on measured optical quantities has been unclear up to now.

In this Letter, we show experimental evidence for the fundamental difference in the creation of $e-h$ pairs between CL and PL signals. We have developed a new method relying on the measurement of the time correlation between the cathodoluminescence emission events from a sample excited by a low intensity fast electrons beam (energy $\approx 60 \mathrm{keV}$ ) in a STEM. We apply it to diamond particles containing a high density of defect centers (nitrogen vacancy, NV). We observed that at low electron currents $(I \leq 100 \mathrm{pA})$, the second order auto-correlation function $\left[g_{\mathrm{CL}}^{(2)}(\tau)=\langle I(t) I(t+\tau)\rangle /\langle I(t)\rangle^{2}\right]$ of the CL signal intensity $I(t)$ displays a large, nanosecond-range peak at zero delay (bunching), which depends on the incoming electron current. This behavior strongly departs from the PL

$g_{\mathrm{PL}}^{(2)}(\tau)$ function which is flat when multiple independent emitters are excited. We show that a model in which plasmons induce a synchronized emission from several emitters can account for this current-intensity-dependent bunching effect. The universality of the phenomemon is exemplified by the observation of $g^{(2)}(0)$ larger than 35 in $\mathrm{CL}$ from defect centers in hexagonal boron nitride $(h$-BN). 


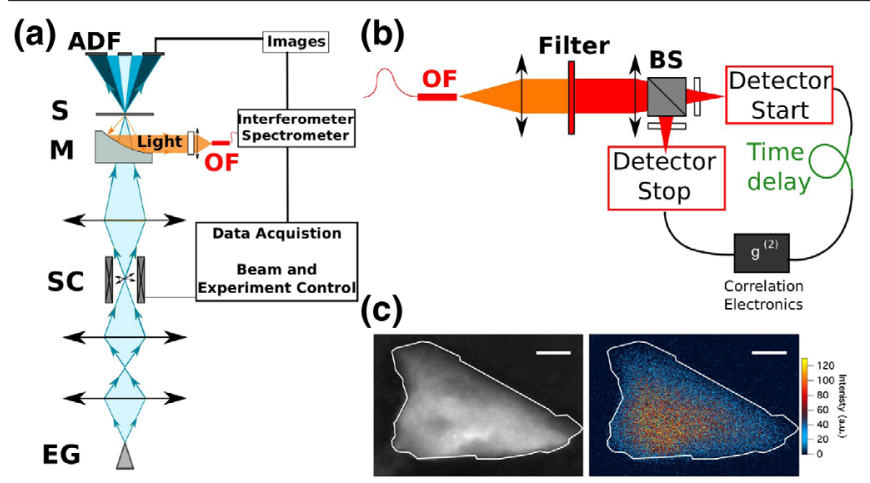

FIG. 1 (color online). CL-STEM setup. (a) A parabolic mirror $(M)$ with a high numerical aperture has been incorporated inside the STEM to collect the CL signal efficiently, forming a collimated beam which is then coupled to a multimode optical fiber (OF). The fiber output beam is sent either to an imaging spectrograph to record the emission spectrum for each pixel, or to a Hanbury Brown-Twiss interferometer for $g_{\mathrm{CL}}^{(2)}(\tau)$ measurement shown in (b). Annular dark field (ADF) detector, sample (S), scanning coils (SC), electron gun (EG). (c) Simultaneously recorded $\mathrm{ADF}$ image (left) and filtered CL maps (right) of the nanodiamond studied in Fig. 2 (scale bar $50 \mathrm{~nm}$ ).

We measured $g_{\mathrm{CL}}^{(2)}(\tau)$ from several diamond nanocrystals [21] (nanodiamond, ND, with a mean size $\approx 100 \mathrm{~nm}$ ) each containing a large number $N \approx 900$ of $N V$ centers [22] using the same setup [Fig. 1(a)] as in Ref. [23]. Briefly, it allows one to measure the $g_{\mathrm{CL}}^{(2)}(\tau)$ with a Hanbury BrownTwiss (HBT) interferometer [Fig. 1(b)] when individual emitters are excited with a nanometric $60 \mathrm{keV}$ electron beam, in conjunction with various other signals, like filtered CL images, CL spectra or the morphological (ADF) images [Fig. 1(c)]. We used a bandpass filter (wavelength range $570-720 \mathrm{~nm}$ ) to collect preferentially the emission of the neutral $\mathrm{NV}^{0}$ center. We chose $\mathrm{NV}$ centers because their photophysical properties are well known, but the observed zero-delay photon bunching is universal, as exemplified on defect centers in $h$-BN (see Supplemental Material for details on these centers [24]).

Figure 2 displays a representative experimental data set. It shows $g_{\mathrm{CL}}^{(2)}(\tau)$ measured at different excitation current intensities (from $I=1$ to $I=10 \mathrm{pA}$; curves with higher excitation currents display the same tendency, see Supplemental Material [24]) with a typical $20 \mathrm{mn}$ integration time. The $g_{\mathrm{CL}}^{(2)}$ function is inferred from the normalization of the time-delay histogram to 1 at long time delays. A bunching $\left[g_{\mathrm{CL}}^{(2)}(0)>1\right]$ peak is observed at all intensities for the studied NDs. The $g_{\mathrm{CL}}^{(2)}(0)$ value increases upon decrease of the electron beam current. In sharp contrast, a $g_{\mathrm{PL}}^{(2)}(\tau)$ taken well below the saturation power in a confocal geometry results in a flat $g_{\mathrm{PL}}^{(2)}(0) \approx 1$ (inset of Fig. 2), as expected, because for a system made up of $N$ emitters, $g_{\mathrm{PL}}^{(2)}(0)=1-1 / N[32] \cdot g_{\mathrm{CL}}^{(2)}(0) \approx 1$ is also retrieved at high excitation current (typically 200 pA, see Supplemental

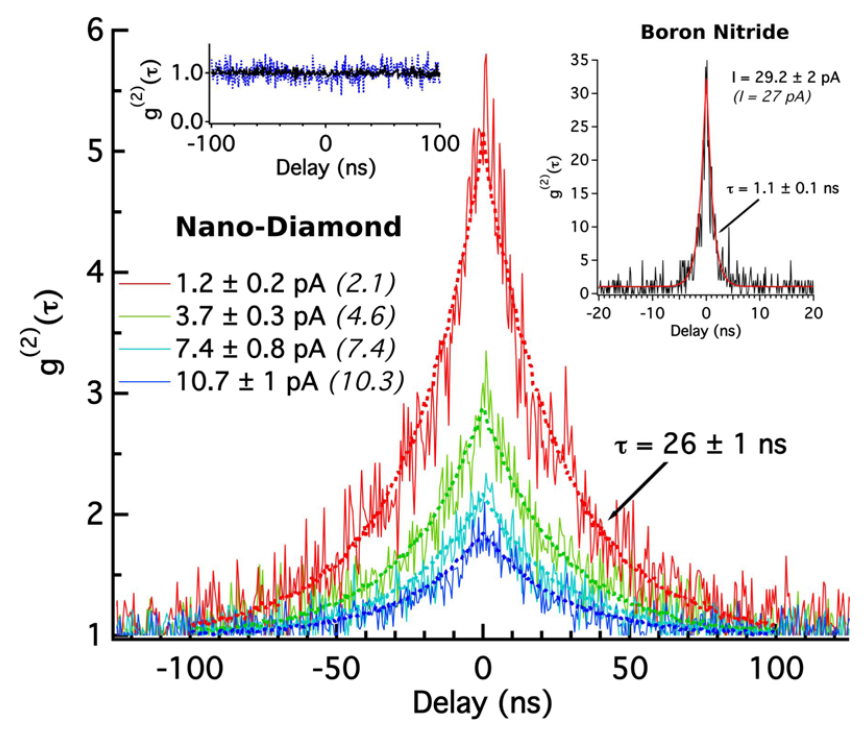

FIG. 2 (color online). $\quad g_{\mathrm{CL}}^{(2)}(\tau)$ measurement (continuous lines) for intensities $I$ ranging from 1.2 to $11 \mathrm{pA}$ for an invidividual ND, $60 \mathrm{~nm}$ thick. Each measurement lasted 5-20 min. The data were fitted with the Monte Carlo simulations explained in the text (dashed line). The lifetime $\tau_{\mathrm{NV}}$ was retrieved using an exponential fit of the bunching curves, leading to $\tau_{\mathrm{NV}} \approx 26 \pm 1 \mathrm{~ns}$. All the parameters of the Monte Carlo simulation were kept fixed except the current, which was changed until a good agreement between the experiment and the simulation was found. The resulting fitted currents are written in parenthesis next to the measured experimental values. Inset, left: PL $g_{P L}^{(2)}(\tau)$ measurements performed at two different laser excitation powers on another ND from the same batch. Inset, right: $g_{\mathrm{CL}}^{(2)}(\tau)$ measurement and Monte Carlo simulation for defect centers in $h$-BN.

Material Fig. 2 [24]). The initial bunching effect with the same peak intensity value can be retrieved by diminishing the electron excitation current again, indicating that the latter effect is reversible. The characteristic time (extracted from an exponential fit, see below) lay between 10 and $30 \mathrm{~ns}$ in all the nearly 100 investigated NDs (26 ns in Fig. 2), which is compatible with the $\mathrm{NV}^{0}$ center excitation lifetime in NDs [33], while for $h$-BN it lay between 1 and 3 ns, which is also compatible with known values [34].

Photon bunching is well known in PL. However, zerodelay bunching appears at the pico-or femtosecond time scales for thermal light [35], amplified spontaneous sources [36], and semiconductor microcavities in the strong coupling regime [37]; it has been also observed at the nanosecond time scale in specific cases (quantum dots in cavities [38] or coupled to plasmons [39], superradiant emitters in cavities [40]). Bunching is also observed in single-emitter fluorescence (organic dyes in particular) due to intersystem crossing from the excited state to metastable states, and in fluorescence correlation spectroscopy due to the fluctuations of the number of emitters in the excitation volume. However, these photon bunchings take place on nonzero delay time scales [41]. Finally, experimental zero-delay bunching larger than 3 has never been reported 
to our knowledge. In summary, all previous reports of photon bunching depart from our observations, which therefore require a different interpretation.

Synchronizing the emission from multiple emitters may lead to bunching. Synchronized emission can happen when two or more centers are excited at the same time. It is worth noting that even for current values corresponding to less than 1 incoming electron per defect excited-state lifetime (typically $I<24 \mathrm{pA}$ for NVs), the bunching effect is present. This clearly indicates that the photon correlations are not related to incoming electron correlations, but are the result of a single electron excitation mechanism.

In the case of a photon exciting a semiconductor, the excitation energy is only slightly larger than that of the emission. A single $e-h$ pair is created, exciting at most one center. In contrast, an electron creates bulk plasmons of high energy ( $E_{p} \approx 30 \mathrm{eV}$ in diamond) [13]. From this, there are two possibilities to explain the bunching. One is the creation of multiple $e-h$ pairs originating from the same plasmon decay, an event made possible thanks to the high energy of the plasmon compared to the gap energy $\left(E_{\mathrm{g}} \approx 5 \mathrm{eV}\right)$. The second is the creation of multiple e-h pairs that may stem from two different plasmons. Whatever the mechanism, all these $e-h$ pairs may excite several centers at once. If at least 2 centers are excited by the recombinations of $e-h$ pairs coming from the same single electron, there is a high probability that at least two photons will be emitted in a given time window. Therefore, each electron produces a packet of photons. If the electrons are sufficiently separated in time, the HBT interferometer will receive well-separated packets of photons, leading to bunching. As the incoming electron current increases, packets of photons produced by different electrons will arrive closer and closer in time until they become indistinguishable. Thus, increasing $I$ will blur the bunching effect. As the electron arrival time statistics are Poissonian, at high $I$, we have $g_{\mathrm{CL}}^{(2)}(\tau) \approx g_{\mathrm{PL}}^{(2)}(\tau)$. The time scale of a packet of photons produced by one electron is approximately the lifetime of the emitters; thus, the width of the bunching peak is related to the emitter lifetime.

Based on this analysis, we developed a Monte Carlo method to calculate the photon emission probability as a function of time for an emitter excited by electrons. We then retrieved the $g_{\mathrm{CL}}^{(2)}(\tau)$ function by calculating the delay between emitted photons. Because the exact number per plasmon and the mechanism of $e-h$ pair production vary in the literature [17,42], we evaluated several models in which we changed the plasmon decay mechanism, with either exactly $n e-h$ pairs produced per plasmon or a Poissonian distribution of $e-h$ pairs with mean value $n$. Taking into account Refs. [17,42] we present results with $n=1$ or 3 , but we obtained similar conclusions for other $n$ values.

In all cases, we define a chain of events made up of (i) the creation of plasmons by an electron and their femtosecond decay into $e-h$ pairs, with probability $P_{\mathrm{el}}^{n}$ [13]:

$$
P_{\mathrm{el}}^{n}\left(L / \lambda_{\mathrm{e}}\right)=\frac{1}{n !}\left(\frac{L}{\lambda_{\mathrm{e}}}\right)^{n} e^{-L / \lambda_{\mathrm{e}}},
$$

where $L / \lambda_{\mathrm{e}}$ is the ratio of the thickness traversed by the electron to its mean free path for plasmon excitation, (ii) the excitation of centers by the $e-h$ pairs happening a few picoseconds later with probability $P_{\text {int }}\left(N, l_{d}, \sigma\right)$, with $l_{d}$ the diffusion length, $\rho$ the defect density considering the material as homogeneous, and $\sigma$ the absorption cross section:

$$
P_{\text {int }}\left(N, l_{d}, \sigma\right)=1-e^{-l_{d} \sigma \rho},
$$

and (iii) the radiative relaxation, at time $t_{\text {rad }}$ for a center (lifetime $\tau_{e}$ ) excited at time $t_{0}$, with probability $P_{\text {rad }}$ :

$$
P_{\text {rad }}\left(t_{\mathrm{rad}}, t_{0}\right)=\frac{1}{\tau_{e}} e^{-\left(t_{\mathrm{rad}}-t_{0}\right) / \tau_{e}} .
$$

In the simulations we consider that the number $N$ of centers likely to absorb an $e$ - $h$ pair is constant, because the number of excited centers is always negligible compared to $N$.

Whatever the parameters, the simulations exhibit a bunching profile converging to 1 at large delays, proving that multiple excitations by a single electron are indeed responsible for the bunching effect. Moreover, they show that the curves can be fitted with an exponential curve, the decay parameter of which is precisely equal to $\tau_{e}$. Finally, they show the negligible influence of the value of $P_{\text {int }}$ over several orders of magnitude, on the value of $g^{(2)}(0)$ (see Supplemental Material [24]), and therefore that the precise values of the parameters $\rho, \sigma$, and $l_{d}$ are not needed to determine $\tau_{e}$ and $g^{(2)}(0)$.

In order to compare the accuracy of the different models (Poissonian or not) we proceeded as follows. First, $\tau_{e}$ was determined by fitting an exponential to an experimental $g_{\mathrm{CL}}^{(2)}(\tau)$ at a given $I . \tau_{e}$ is then kept fixed for the curves acquired for other $I . L / \lambda_{e}$ is directly measured from EELS data (see Supplemental Material [24]). The current is then determined as the only free parameter (we took $\rho$ from Ref. [22], $\sigma \approx 95 \mathrm{~nm}^{2}$ [43], and $l_{d} \approx 50 \mathrm{~nm}$ for NDs [44]) by comparing a series of simulations to experimental curves. The accuracy of the different models can thus be inferred from the comparison between the fitted and simulated results.

As seen in Fig. 3, all the simulations reproduce the bunching effect, again confirming that multiple excitations by a single electron are responsible for the effect. Models in which the number of $e-h$ pairs per plasmon is constant underestimate the bunching effect. Models assuming a Poissonian distribution for the plasmon decay seem to work better, highlighting the complexity of plasmon decay in the materials, but without giving any reason or insight into it. It may be due to stochastic phonon-e- $h$ interaction, or to the creation of Auger and secondary electrons for example. Further studies, outside the scope of the present Letter, would be needed to clarify the exact plasmon decay mechanisms. In contrast with PL, the bunching effect is thus intrinsic to CL physics and needs to be taken into account when performing HBT experiments in STEM. 


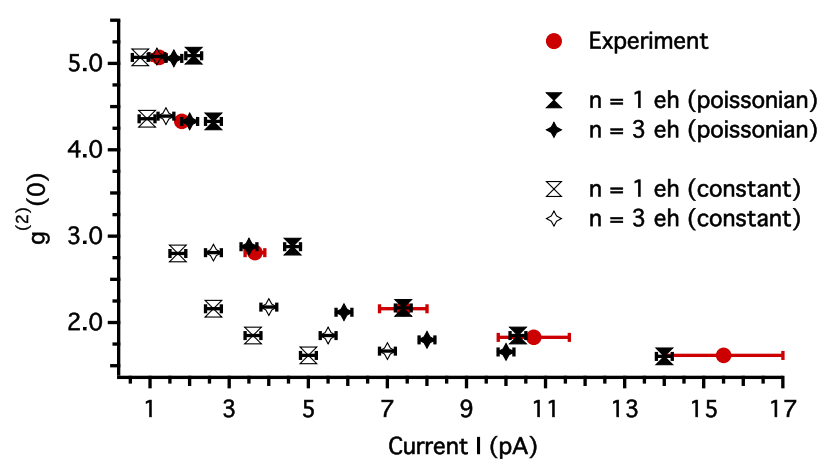

FIG. 3 (color online). $g_{\mathrm{CL}}^{(2)}(0)$ calculated from Monte Carlo simulations within the two different models for the decay mechanism (see text) compared to experimental data points extracted from Fig. 2. For all the simulations, all parameters are fixed except the current. Error bars in the simulations are generated by introducing a $\pm 1 \mathrm{~ns}$ error in the estimate of $\tau_{e}$ and a very conservative $\pm 10 \%$ error on $L / \lambda_{e}$. The bunching effect is qualitatively reproduced by both models.

Finally, we turn to the low $N$ regime, in which antibunching [23] and bunching compete. We modeled phenomenologically the behavior of the $g_{\mathrm{CL}}^{(2)}(\tau)$ function for a small number of centers $(N<5)$ and a thickness of $30 \mathrm{~nm}$, which means that the number of plasmons per electron can be considered to be equal to 1 . The equation after simplification is given by (see details in the Supplemental Material [24])

$$
g^{(2)}(\tau)=\frac{I_{0}}{I \times P_{\mathrm{el}}^{1}}\left(1-\frac{1}{N}\right) e^{-\tau / \tau_{e}}+\left(1-\frac{1}{N} e^{-\tau / \tau_{e}}\right),
$$

where $I_{0}$ is the incoming electron current required to have one electron per lifetime. The first term in Eq. (4) represents correlations due to electron excitation and thus is responsible for the bunching effect. The second term, identical to the $g_{P L}^{(2)}(\tau)$ expression, represents the correlation due to the sample emission and is responsible for the antibunching behavior for single photon emitters. We can clearly see that when the current increases, the bunching part is blurred, leading to a $g^{(2)}(\tau)$ function similar to that for PL [45]. We also note that the expression formally diverges at zero current. However, at such a value, the bunch of photons is emitted with an infinite delay, preventing such a diverging limit from being reached experimentally. Also, Eq. (4) indicates the possibility of getting large $g^{(2)}(0)$ for short lifetimes (large $I_{0}$ ), as clearly demonstrated for defect centers in $h$-BN (Fig. 2). The $g_{\mathrm{CL}}^{(2)}(\tau)$ for $N=1$ and $N=2$ are shown in Fig. 4 for different currents. An individual NV center in a particle can only be excited by one $e-h$ pair at a time, leading to antibunching with $g_{\mathrm{CL}}^{(2)}(0)=0$ for all currents. If more than one center is present, bunching effects will appear superimposed on the antibunching behavior. For $N=2$, a transition from bunching to antibunching is expected upon increasing $I$. This shows that the law

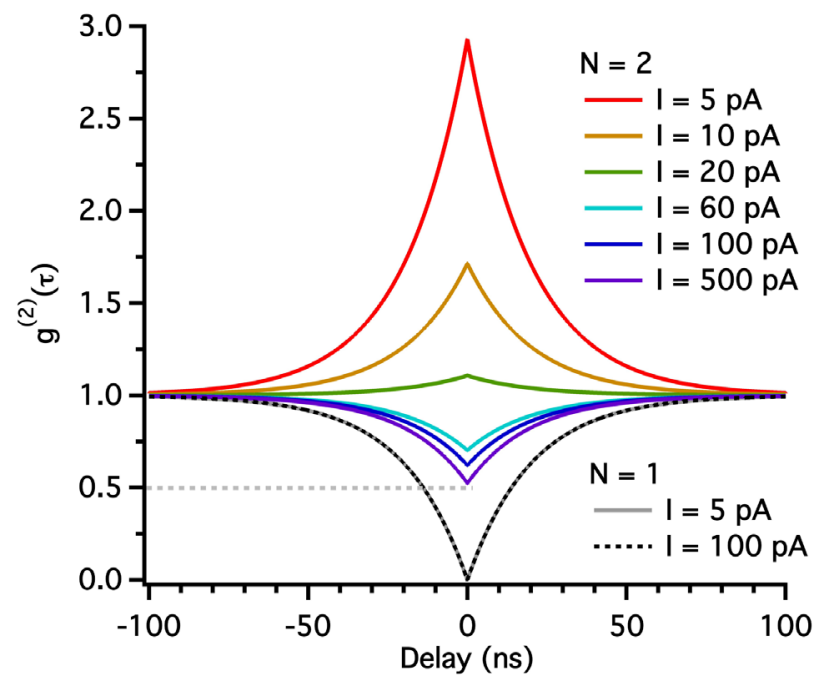

FIG. 4 (color online). Simulation for a ND with $L=30 \mathrm{~nm}$ and $\tau_{e}=20$ ns. For $N=1$ the $g_{\mathrm{CL}}^{(2)}(\tau)$ is independent of the probe current. For $N>1$ (here $N=2$ ) the expected antibunching behavior is retrieved at high current but not at low current.

$g^{(2)}(0)=1-1 / N$ is difficult to observe in CL-STEM. The transition between the bunching and antibunching is also difficult to observe experimentally, as the necessary integration time to obtain a meaningful $g_{\mathrm{CL}}^{(2)}(\tau)$ (typically $5 \mathrm{~min}$ ) is large enough to induce radiation damage for a small number of defect centers.

In conclusion, we have shown that $g_{\mathrm{CL}}^{(2)}(\tau)$ of defect centers in two examples of wide band-gap semiconductors exhibits a bunching behavior depending on the excitation current and the lifetime of the emitter. This phenomenon has been shown to arise from the synchronization of multiple excitations via primary excitation decay into multiple $e-h$ pairs and should be observable in any luminescent system by CL. Moreover, we believe that the autocorrelation shape with a single peak and a large $g^{(2)}(0) \gg 2$ could be recovered with an optical excitation, if emitters are excited with intense short laser pulses which are randomly separated in time. The ability to model the emitter lifetime with a simple exponential fit opens the way to easy and quantitative measurement of any emitter lifetime with a spatial resolution only limited by $e-h$ pair diffusion lengths [46] and with a high signal-to-noise ratio. Finally, the high observed $g^{(2)}(0)$ value is independent of the emission wavelength (tunable by material choice), meaning such photon sources could be of interest in two-photon excitation fluorescence [47], where the amplitude of the effect depends linearly on $g^{(2)}(0)$.

This work has received support from the French State through the National Agency for Research under the program of future investment EQUIPEX TEMPOSCHROMATEM with the reference ANR-10-EQPX-50 as well as the French General Directorate for Armament and the Labex NanoSaclay under the name BioCL. 
The research leading to these results has received funding from the European Union Seventh Framework Programme [No. FP7/2007- 2013] under Grant Agreement No. n312483 (ESTEEM2). We thank M. Walls for his in-depth reading of the manuscript and A. Zobelli for fruitful discussions. S.M. and M. K. want to thank Professor J. Garcia de Abajo for pointing out the interest of highly bunched statistics for two-photon excitations.

*mathieu.kociak@u-psud.fr

[1] F. J. Garcia de Abajo and M. Kociak, New J. Phys. 10, 073035 (2008).

[2] E. J. R. Vesseur, R. de Waele, M. Kuttge, and A. Polman, Nano Lett. 7, 2843 (2007).

[3] J. Nelayah, M. Kociak, O. Stéphan, F. J. Garcia de Abajo, M. Tencé, L. Henrard, D. Taverna, I. Pastoriza-Santos, L. M. Liz-Marzan, and C. Colliex, Nat. Phys. 3, 348 (2007).

[4] M. Bosman, V. J. Keast, M. Watanabe, A. I. Maaroof, and M. B. Cortie, Nanotechnology 18, 165505 (2007).

[5] F. J. Garcia de Abajo and M. Kociak, Phys. Rev. Lett. 100, 106804 (2008).

[6] U. Hohenester, H. Ditlbacher, and J. R. Krenn, Phys. Rev. Lett. 103, 106801 (2009).

[7] B. Yacobi and D. Holt, Cathodoluminescence Microscopy of Inorganic Solids (Springer, New York, 1990).

[8] M. Kociak, O. Stéphan, A. Gloter, L. F.Zagonel, L. H. G. Tizei, M. Tencé, K. March, J.-D. Blazit, Z. Mahfoud, A. Losquin, S. Meuret, and C. Colliex, C.R. Phys. 15, 158 (2014).

[9] M. Grundmann, O. Stier, and D. Bimberg, Phys. Rev. B 52, 11969 (1995).

[10] J. Garayt, J. Gérard, F. Enjalbert, L. Ferlazzo, S. Founta, E. Martinez-Guerrero, F. Rol, D. Araujo, R. Cox, B. Daudin, B. Gayral, L. Dang, and H. Mariette, Physica (Amsterdam) 26E, 203 (2005).

[11] S. Lim, M. Brewster, F. Qian, Y. Li, C. M. Lieber, and S. Gradecak, Nano Lett. 9, 3940 (2009).

[12] L. F. Zagonel, S. Mazzucco, M. Tencé, K. March, R. Bernard, B. Laslier, G. Jacopin, M. Tchernycheva, L. Rigutti, F. H. Julien, R. Songmuang, and M. Kociak, Nano Lett. 11, 568 (2011).

[13] R. Egerton, EELS in the Electron Microscope (Springer, New York, 1996).

[14] W. Shockley, Solid State Electron. 2, 35 (1961).

[15] L. Campbell, F. Gao, R. Devanathan, and W. J. Weber, Nucl. Instrum. Methods Phys. Res., Sect. A 579, 454 (2007).

[16] D. Pines, Rev. Mod. Phys. 28, 184 (1956).

[17] A. Rothwarf, J. Appl. Phys. 44, 752 (1973).

[18] C. A. Klein, J. Appl. Phys. 39, 2029 (1968).

[19] P. A. Rodnyi, P. Dorenbos, and C. W. E. van Eijk, MRS Online Proc. Libr. 348, 379 (1994).

[20] Z. Mahfoud, A. T. Dijksman, C. Javaux, P. Bassoul, A.-L. Baudrion, J. Plain, B. Dubertret, and M. Kociak, J. Phys. Chem. Lett. 4, 4090 (2013).

[21] L.-J. Su, C.-Y. Fang, Y.-T. Chang, K.-M. Chen, Y.-C. Yu, J.-H. Hsu, and H.-C. Chang, Nanotechnology 24, 315702 (2013).

[22] J. Botsoa, T. Sauvage, M.-P. Adam, P. Desgardin, E. Leoni, B. Courtois, F. Treussart, and M.-F. Barthe, Phys. Rev. B 84, 125209 (2011).
[23] L. H. G. Tizei and M. Kociak, Phys. Rev. Lett. 110, 153604 (2013).

[24] See Supplemental Material at http://link.aps.org/ supplemental/10.1103/PhysRevLett.114.197401 for additional experiments on nanodiamonds, information on the $\mathrm{hBN}$ defects, description of the thickness to mean free path measurements, details on Monte Carlo simulations and analytical models, which includes Refs. [25-31].

[25] Y. Hernandez, V. Nicolosi, M. Lotya, F. M. Blighe, Z. Sun, S. De, I. T. McGovern, B. Holland, M. Byrne, Y. K. Gun'Ko, J. J. Boland, P. Niraj, G. Duesberg, S. Krishnamurthy, R. Goodhue, J. Hutchison, V. Scardaci, A. C. Ferrari, and J. N. Coleman, Nat. Nanotechnol. 3, 563 (2008).

[26] N. Alem, R. Erni, C. Kisielowski, M. D. Rossell, W. Gannett, and A. Zettl, Phys. Rev. B 80, 155425 (2009).

[27] A. L. Gibb, N. Alem, J.-H. Chen, K. J. Erickson, J. Ciston, A. Gautam, M. Linck, and A. Zettl, J. Am. Chem. Soc. 135, 6758 (2013).

[28] N. Alem, Q. M. Ramasse, C. R. Seabourne, O. V. Yazyev, K. Erickson, M. C. Sarahan, C. Kisielowski, A. J. Scott, S. G. Louie, and A. Zettl, Phys. Rev. Lett. 109, 205502 (2012).

[29] J. H. Warner, M.H. Rümmeli, A. Bachmatiuk, and B. Büchner, ACS Nano 4, 1299 (2010).

[30] K. Hara, X. Liu, M. Yamauchi, Y. Kawanishi, H. Kominami, and Y. Nakanishi, Phys. Status Solidi C 8, 2509 (2011).

[31] L. Museur, D. Anglos, J.-P. Petitet, J.-P. Michel, and A. V. Kanaev, J. Lumin. 127, 595 (2007).

[32] M. Fox, Quantum Optics: An Introduction (Oxford Master Series in Physics, New York, 2006).

[33] G. Liaugaudas, G. Davies, K. Suhling, R. U. A. Khan, and D. J. F. Evans, J. Phys. Condens. Matter 24, 435503 (2012).

[34] L. Museur, E. Feldbach, and A. Kanaev, Phys. Rev. B 78, 155204 (2008).

[35] B. L. Morgan and L. Mandel, Phys. Rev. Lett. 16, 1012 (1966).

[36] M. Blazek, S. Hartmann, A. Molitor, and W. Elsaesser, Opt. Lett. 36, 3455 (2011).

[37] D. W. Snoke, Phys. Status Solidi B 238, 389 (2003).

[38] M. Winger, T. Volz, G. Tarel, S. Portolan, A. Badolato, K. J. Hennessy, E. L. Hu, A. Beveratos, J. Finley, V. Savona, and A. M. C. Imamoğlu, Phys. Rev. Lett. 103, 207403 (2009).

[39] Y.-S. Park, Y. Ghosh, Y. Chen, A. Piryatinski, P. Xu, N. H. Mack, H.-L. Wang, V. I. Klimov, J. A. Hollingsworth, and H. Htoon, Phys. Rev. Lett. 110, 117401 (2013).

[40] A. Auffèves, D. Gerace, S. Portolan, A. Drezet, and M. França Santos, New J. Phys. 13, 093020 (2011).

[41] S. C. Kitson, P. Jonsson, J. G. Rarity, and P. R. Tapster, Phys. Rev. A 58, 620 (1998).

[42] C. A. Klein, J. Phys. Soc. Jpn. Suppl. 21, 307 (1966).

[43] R. Chapman and T. Plakhotnik, Chem. Phys. Lett. 507, 190 (2011).

[44] L. H. G. Tizei and M. Kociak, Nanotechnology 23, 175702 (2012).

[45] A. Beveratos, R. Brouri, J. Poizat, and P. Grangier, $Q C M C$ 3 Proceedings (2001).

[46] L. F. Zagonel, L. Rigutti, M. Tchernycheva, G. Jacopin, R. Songmuang, and M. Kociak, Nanotechnology 23, 455205 (2012).

[47] A. Jechow, M. Seefeldt, H. Kurzke, A. Heuer, and R. Menzel, Nat. Photonics 7, 973 (2013). 